\title{
Exploring Information Spaces by Using Tangible Magic Lenses in a Tabletop Environment
}

\section{Martin Spindler}

Otto-von-Guericke University

Department of Computer Science

Universitätsplatz 2

Magdeburg, 39106 Germany

martin.spindler@ovgu.de

\section{Raimund Dachselt}

Otto-von-Guericke University

Department of Computer Science

Universitätsplatz 2

Magdeburg, 39106 Germany

dachselt@acm.org

\begin{abstract}
To solve the challenge of exploring large information spaces on interactive surfaces such as tabletops, we developed an optically tracked, lightweight, passive display (magic lens) that provides elegant three-dimensional exploration of rich datasets. This can either be volumetric, layered, zoomable, or temporal information spaces, which are mapped onto the physical volume above a tabletop. By moving the magic lens through the volume, corresponding data is displayed, thus serving as a window into virtuality. Hereby, various interaction techniques are introduced, which especially utilize the lens' height above a tabletop in a novel way, e.g. for zooming or displaying information layers.
\end{abstract}

\section{Keywords}

Tangible Magic Lens, 3D Navigation, Data Exploration, Natural Interaction, Passive Display, IR-Tracking

\section{ACM Classification Keywords}

H5.2. Information interfaces and presentation (e.g., $\mathrm{HCI}$ ): User Interfaces - Input devices and strategies; Interaction Styles

\section{General Terms}

Design, Human Factors 

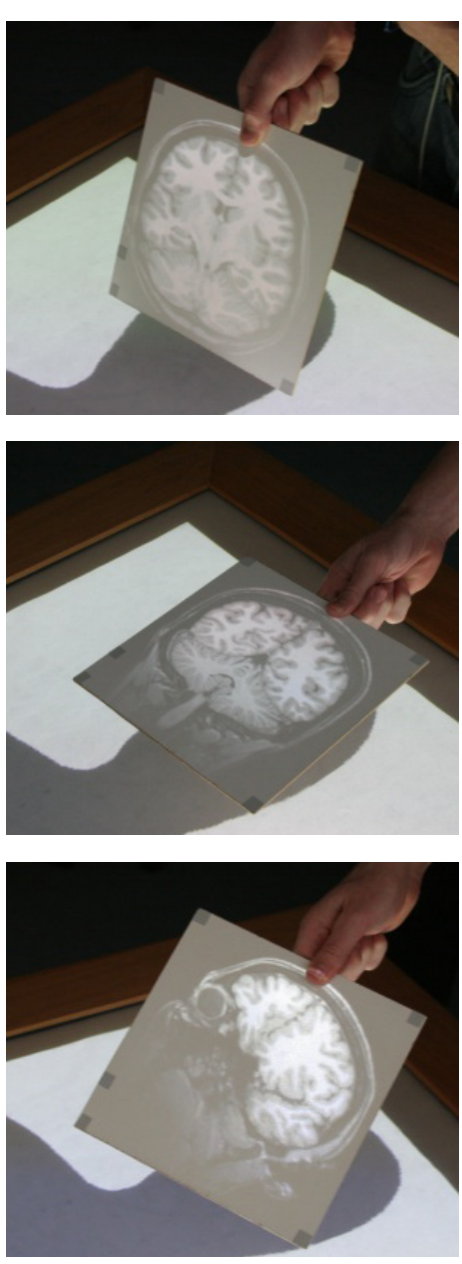

Figure 1. Users can slice through volumetric information spaces (e.g. an MRI scan of a human head) by arbitrarily moving or tilting the magic lens in the physical 3D space above a tabletop.

\section{Introduction}

Despite rapid improvements in research and technology for large displays and interactive surfaces over the past decade, the physical 3D space above interactive tabletops is still being used rather scarcely. This is because interaction techniques mostly focus on the tabletop surface itself. At the same time, information spaces are growing continuously and thus data filtering and exploration tasks become more and more challenging. In this respect, detail and context techniques, such as the concept of Magic Lenses, are a promising solution.

With PaperLens [9], we introduced a lightweight and robust handheld display solution that allows the exploration of various information spaces above a tabletop. This was accomplished by explicitly utilizing the Z-axis (height above the surface) as an interaction metaphor. Depending on the nature of the displayed data, we distinguish between four spaces: the volumetric, layered, zoomable, and temporal information space. By moving a tracked sheet of paper through the interaction volume, the displayed content adapts according to the current position with regard to the contextual tabletop display.

Although the idea of tangible magic lenses and the interaction above a surface is not entirely new, novel technological solutions such as [5] and [6] suggest the strong potential of this research area. In summary, our main contributions are (I) a technical solution for a lightweight and untethered tangible lens in a tabletop environment, (II) the introduction of layered and temporal information spaces in combination with mobile lenses, and (III) a systematic classification of different data types and how they might be effectively explored with movable lenses.

\section{Related Work}

The Magic Lens concept [1] for presenting customized views of an underlying application has been a field of research for many years now. Over the years, this idea was extended to tangible magic lenses [10] for the application with tabletop environments.

Various tangible magic lens prototypes have been developed for spatially aware displays, which differ in their implementation and application. One of the first prototypes has been published by Fitzmaurice [2], who presented a spatially aware palmtop computer for exploring 3D-situated information spaces for revealing virtual information associated with real items. Other examples are Peephole Display by Yee [11] for exploring 2D virtual workspaces and the metaDESK system by Ullmer and Ishii [10] for navigating in 3D geo spaces. Sanneblad and Holmquist use small active displays (tablet PCs) in combination with large screens for navigating through 2D geographical maps and for providing detail and annotation capabilities [7]. However, such systems usually only focus on certain aspects of spatial data exploration, rarely use the physical volume above a display and often employ complicated, expensive or heavy hardware such as tablet PCs.

In terms of display approach, a general distinction can be made between active (UMPCs or tablet PCs) and passive (projection screen) lenses. Usually, passive lenses, such as paper displays, are more lightweight, flexible, robust and variable in shape as compared to their active counterparts. For PaperWindows, Holman et al. [4] used a top-projector to project a classic WIMP/ desktop environment onto a spatially aware piece of paper. 

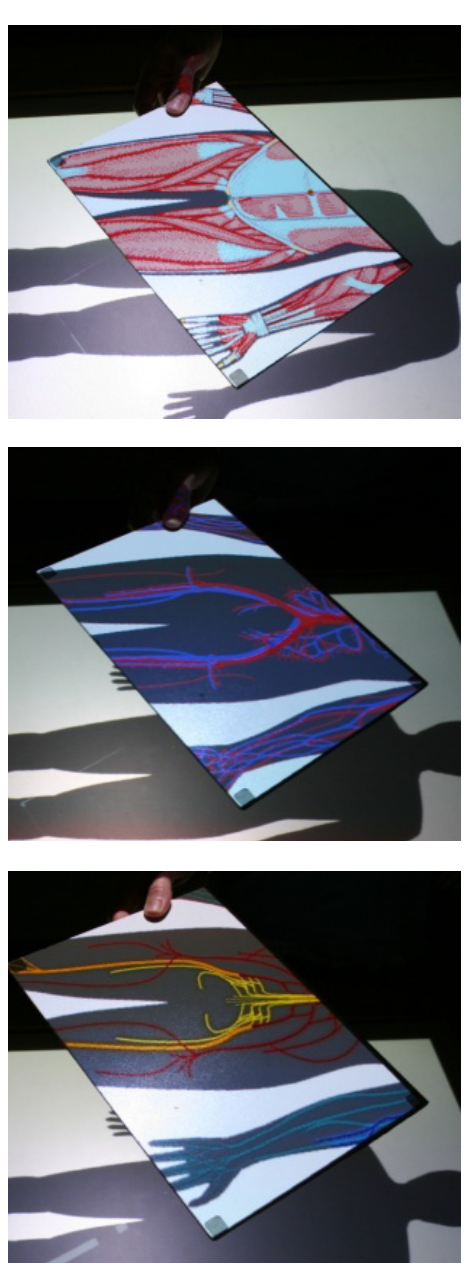

Figure 3. Various layers of information, in this case

representing different human body systems, can be chosen from by lifting and lowering the magic lens.

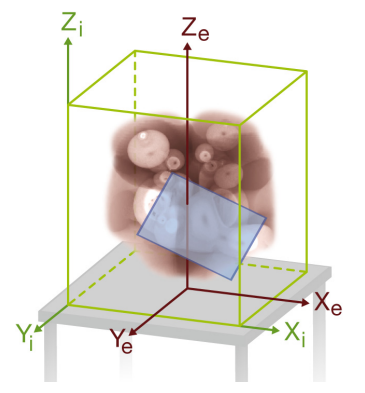

(a) Volumetric Info Space

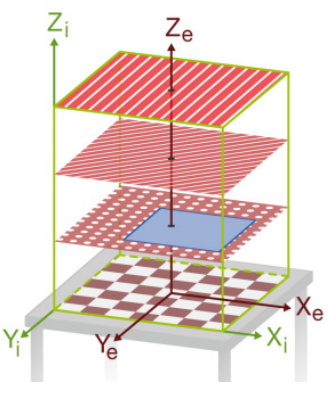

(b) Layered Info Space

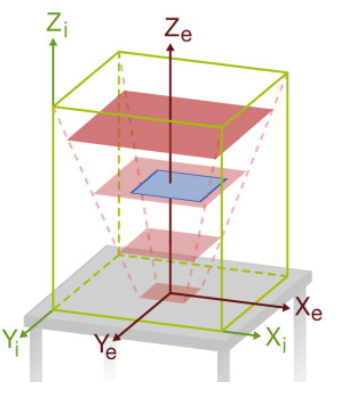

(c) Zoomable Info Space

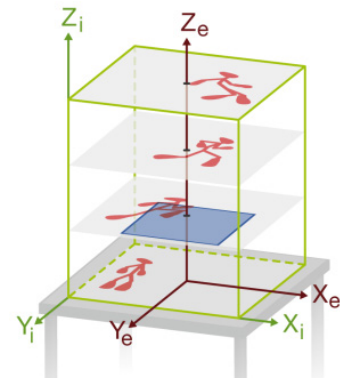

(d) Temporal Info Space

Figure 2: By moving the PaperLens (blue rectangle) through the physical 3D space above the tabletop, i.e. the interaction space $\left(X_{i} Y_{i} Z_{i}\right.$, green), users can examine four different types of explorable information spaces $\left(X_{e} Y_{e} Z_{e}, r e d\right)$.

\section{Exploring Information Spaces}

In this section, PaperLenses are presented as spatially aware tangible displays that are suitable for exploring virtual information spaces in a more natural manner. Similar to a suggestion of Fitzmaurice [2], the principle goal was to find a set of meaningful mappings that translates the movement of a PaperLens in real world space above a tabletop (the interaction space) into an intended action in a virtual information space (the exploration space).

With the exploration space a virtual 3D volumetric space is described that is virtually "filled" with the data the user intends to explore. According to the type and extent of the data, four different classes of exploration spaces can be distinguished: volumetric, layered, zoomable, and temporal information spaces. In order to let users spatially explore these classes in a more natural way, appropriate mappings from the interaction space $\left(X_{i} Y_{i} Z_{i}\right)$ onto the exploration space coordinate system $\left(X_{e} Y_{e} Z_{e}\right)$ needs to be found. Hereby, all four classes share a similar mapping in $\mathrm{X}_{\mathrm{e}} \mathrm{Y}_{\mathrm{e}}$-space, but differ conceptually in the third dimension ( $Z_{\mathrm{e}}$-axis). In the following, each class and its corresponding mappings will be discussed in more detail.

\section{Volumetric Information Space}

The volumetric information space is a set of 3D samples (voxels) with a volumetric nature. Typical examples are datasets acquired via CT or MRI techniques used in the realm of medical or scientific visualization. Volumetric data exhibit a continuous form in all three dimensions and thus allow for a direct linear mapping from $\left(X_{i} Y_{i} Z_{i}\right)$ onto $\left(X_{e} Y_{e} Z_{e}\right)$, see Figure $2 a$.

Figure 1 shows a prototype that allows users to slice through an MRI scan of a human head by arbitrarily moving and tilting the lens above a tabletop.

\section{Layered Information Space}

The layered information space can be defined as a set of 2D information layers (raster data and/or vector 

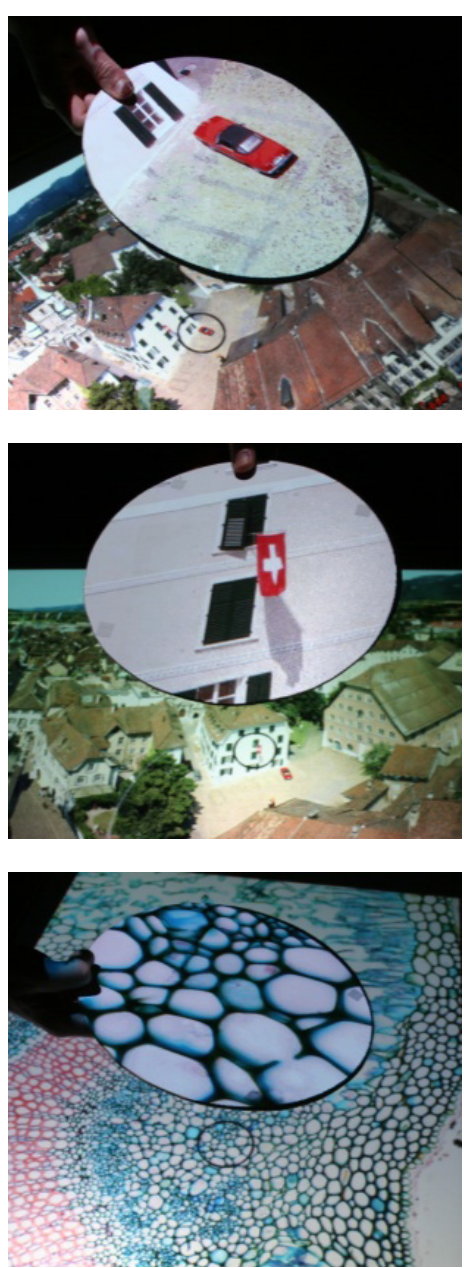

Figure 4. Users can zoom in/out by lifting or lowering the lens above a tabletop. A projected contour line of the lens on the tabletop visualizes the lens' current position within the image. data) with each layer representing a unique feature of a model. Layered information spaces are commonly employed in various application fields, such as geographic information systems or interactive maps. In general, these spaces are continuous within a 2D plane and thus allow for a direct linear mapping from the $X_{i} Y_{i}$ space onto the $X_{\mathrm{e}} \mathrm{Y}_{\mathrm{e}}$-space (see Figure $2 b$ ). The mapping from $Z_{i}$ to $Z_{e}$ is not as straightforward. By dividing the $Z_{\mathrm{e}}$-axis into discreet intervals and by associating these intervals with distinct layers, a "volumetric" layered information space is constructed with each layer covering a distinct "height" in the exploration space - that can be mapped directly onto the interaction space.

Figure 3 shows a prototype that allows medical students to examine four information layers: the skeletal, muscular, blood, and nervous systems of the human body. They can be examined by lifting/lowering the lens (layer selection) or by horizontally moving the lens (navigation within a layer).

\section{Zoomable Information Space}

With zoomable information spaces, large continuous 2D information worlds (e.g. gigapixel images or maps) can be represented that extend far beyond the tabletop in their original size. In this context, zooming and panning usually play an essential role. The concept of spacescale diagrams [4] is a possible way to describe such spaces. It employs a pyramidal representation of the information world with the pyramid's height representing the level of detail/zoom. This enables the direct mapping from $\left(X_{i} Y_{i} Z_{i}\right)$-space onto the $\left(X_{e} Y_{e} Z_{e}\right)$ space in such a way that the pyramid's $Z_{\mathrm{e}}$-axis is aligned with the $Z_{i}$-axis (see Figure $2 c$ ). By this means, depth translation can be interpreted as a scaling function that allows users to zoom into the information world by lifting the lens. This metaphor is similar to the real world experience of many users who bring objects of interest closer to their eyes in order to examine them in more detail. At the same time, panning operations can be expressed by means of XYtranslation.

Figure 4 shows a prototype that allows the exploration of zoomable information spaces. Here, further details are revealed from high resolution photographs when users move the lens up.

\section{Temporal Information Space}

Temporal information spaces are a concept for describing 2D time-dependent data, such as video sequences. As illustrated in Figure $2 \mathrm{~d}$, this is achieved by virtually stacking layers of time (or time frames) one upon the other, in order to create a volumetric description $\left(\mathrm{X}_{\mathrm{e}} \mathrm{Y}_{\mathrm{e}} \mathrm{Z}_{\mathrm{e}}\right)$ of the temporal data with the $\mathrm{Z}_{\mathrm{e}}$ axis representing the time or "state". This volume can then easily be mapped onto the interaction space

$\left(\mathrm{X}_{\mathrm{i}} \mathrm{Y}_{\mathrm{i}} \mathrm{Z}_{\mathrm{i}}\right)$, which allows users to navigate through time by lifting/lowering the lens (depth translation).

Additionally, the tabletop can supply a collection of several temporal datasets (e.g. video snippets) that are "scattered" over the display (each one represented by a still image). This enables users to select a piece of temporal data by simply moving the lens over it (XYtranslation).

Figure 7 shows a prototypic video snippet browser. Here, two short video sequences (represented as still images) are arranged on the tabletop display that can be selected and "watched" as described earlier. 

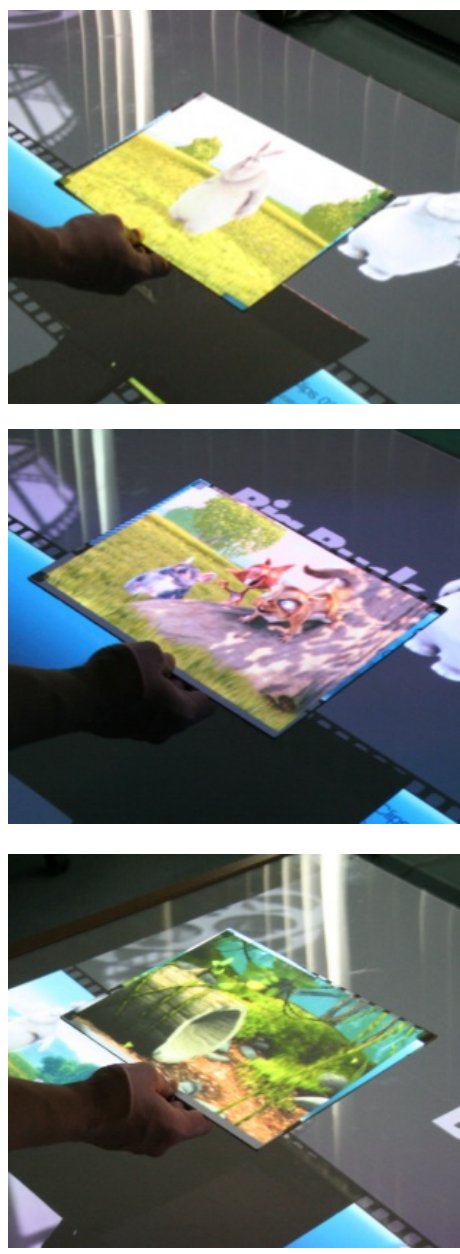

Figure 7. By moving the magic lens up and down, users can "watch" videos. In this way, they can easily perform basic operations such as going forward or backward in time in slow or fast motion.

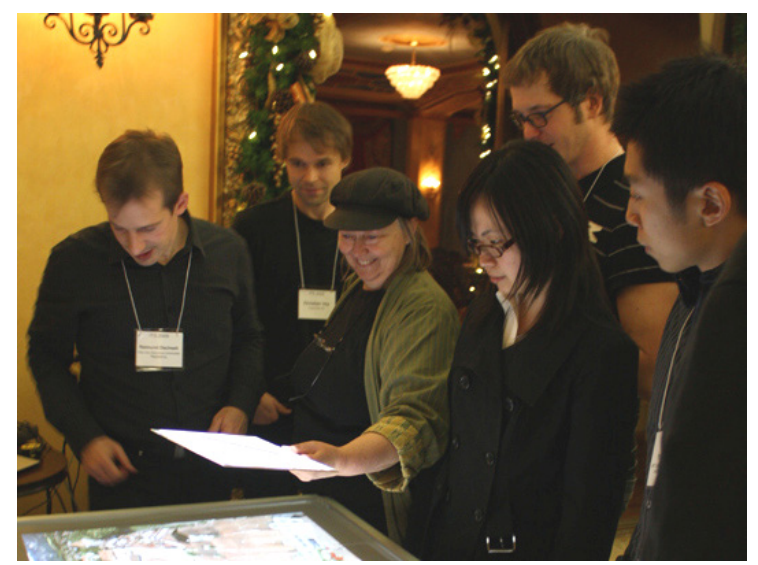

Figure 5. Demoing PaperLens at ITS2009 in Banff, Canada.

\section{Technical Approach}

As illustrated in Figure 6, the general setup of our system consists of a back-projected tabletop, the PaperLens (not visible in Figure 6), and a top-projector and infrared (IR) camera both mounted onto the ceiling. The PaperLens comes in different shapes, such as rectangle, squares or circles, made of pressboard or Plexiglas. The typical size of a PaperLens is in the range of 20 to $40 \mathrm{~cm}$. For tracking, we attached several IRreflective markers $(5 \times 5 \mathrm{~mm})$ onto the lens corners/ borders. The tracking was implemented via Natural Point's OptiTrack Tracking Tools 2.0 with six IR cameras at $50 \mathrm{~Hz}$. Once the position and orientation of a lens is known, the ceiling-mounted top-projector can be used to project image content onto the paper lens display (with perspective correction support).

\section{Presentation History}

The implementation of the magic lens system started at the end of 2008 and the system considerably improved since then. The first prototype as well as a formative user study was published in [1]. A further developed concept of the magic lens system was published as a full paper [9] at the annual conference on Interactive Tabletops and Surfaces (ACM ITS2009). We also gave a couple of demos at the local university usually within the scope of public events or during lab presentations. All presentations have been perceived very well by the audience (approx. 200 German visitors, including children). Furthermore, we demoed our magic lens system at ITS2009 in Banff, Canada, where we received very positive feedback from the $\mathrm{HCI} / \mathrm{Tabletop}$ community (see Figure 5).

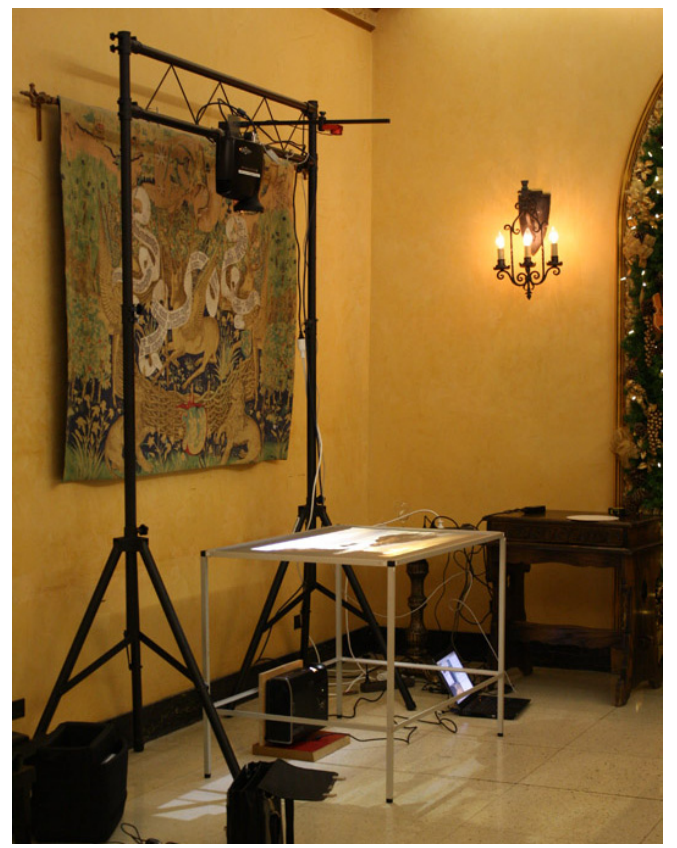

Figure 6. Demo setup of PaperLens at ITS2009 in Banff. 


\section{Conclusion and Future Work}

With PaperLens we presented a spatially aware handheld lens. It is a lightweight, flexible, shape-variable and robust display solution that can be used to explore four different types of data spaces: the volumetric, layered, zoomable, and temporal information space. The PaperLens serves as a window into these virtual spaces. The interaction is accomplished by moving the lens in the physical 3D space above a tabletop.

Thereby, we focused on employing the Z-dimension (height above the tabletop) in order to develop a set of natural lens navigation techniques. We have implemented prototypes for all of the four classes of information spaces. More information about PaperLens can be found in [9].

\section{Acknowledgements}

We like to express our special gratitude to Jana Sieber for her initial implementation. We also thank Lars Uebernickel, Ricardo Langner, and Michel Hauschild for their great support. This work was funded by the "Stifterverband für die Deutsche Wissenschaft" and the German Ministry of Education and Science (BMBF) within the ViERforES project (no. 01IM08003C).

\section{References}

[1] E. A. Bier, M. C. Stone, K. Pier, W. Buxton, and T. $D$. Derose. Toolglass and magic lenses: The seethrough interface, In Proc. of SIGGRAPH '93, 1993, pp. 73-80.

[2] G. W. Fitzmaurice, "Situated information spaces and spatially aware palmtop computers", Commun. ACM, 36(7), 1993, pp. 39-49.

[3] G. W. Furnas and B. B. Bederson, "Space-Scale Diagrams: Understanding Multiscale Interfaces", In
Proc. of CHI '95, ACM Press, New York, USA, 1995, pp. 234-241.

[4] D. Holman, R. Vertegaal, M. Altosaar, N. Troje, and D. Johns, "Paper Windows: Interaction Techniques for Digital Paper", In Proc. of CHI '05, ACM, New York, NY, USA, 2005, pp. 591-599.

[5] S. Izadi, S. Hodges, S. Taylor, D. Rosenfeld, N. Villar, A. Butler, and J. Westhues, "Going Beyond the Display: A Surface Technology with an Electronically Switchable Diffuser", In Proc. of UIST '08, ACM, New York, USA, 2008, pp. 269-278.

[6] Y. Kakehi and T. Naemura, "UlteriorScape: Interactive optical superimposition on a viewdependent tabletop display", In Proc. of 3rd IEEE International Workshop on Horizontal Interactive Human Computer Systems TABLETOP 2008, 2008, pp. 189-192.

[7] J. Sanneblad and L. E. Holmquist, "Ubiquitous graphics: combining hand-held and wall-size displays to interact with large images", In Proc. of AVI '06, ACM, New York, NY, USA, 2006, pp. 373-377.

[8] M. Spindler, J. Sieber, and R. Dachselt, "Using Spatially Aware Tangible Displays for Exploring Virtual Spaces". In Proceedings of Mensch und Computer 2009. Oldenbourg Publishing, Berlin, Germany 2009, 253-262.

[9] M. Spindler, S. Stellmach, and R. Dachselt, "PaperLens: Advanced Magic Lens Interaction above the Tabletop". In Proceedings of Interactive Tabletops and Surfaces 2009. Banff, Canada, November 2009, To be published.

[10] B. Ullmer and H. Ishii, "The metaDESK: Models and prototypes for tangible user interfaces", In Proc. of

UIST '97, ACM, New York, NY, USA, 1997, pp. 223-232.

[11] K.-P. Yee, "Peephole displays: pen interaction on spatially aware handheld computers", In Proc. of CHI '03, ACM, New York, USA, 2003, pp. 1-8. 